\title{
MAGNETIC EQUILIBRIA AND INSTABILITIES
}

\author{
TAKASH I SAKURAI
}

National Astronomical Observatory, Mitaka, Tokyo, Japan

\begin{abstract}
Solar flares are understood as a process of explosive liberation of magnetic energy, coming after a slow phase of energy build-up. The slow evolution of magnetic equilibria may end up with (a) the termination of an equilibrium sequence, or (b) an instability. The distinction between the two can be made by drawing schematic potential curves. Case (a) has been extensively studied in two-dimensional models. The appearance of multiple solutions, or disappearance of a solution takes place as the system evolves away from the current-free configuration. Case (b) can be discussed in terms of ideal MHD or resistive MHD instabilities. A possible route to explosive energy release is suggested by combining these two cases.
\end{abstract}

\section{Introduction}

This review addresses theoretical aspects of energy build-up and energy liberation processes in solar flares. Solar flares take place in the solar corona above active regions. The heating of the corona from its quiescent $2 \mathrm{MK}$ state to a 20-30 MK flare state indicates that the density of energy which produces flares must greatly exceed the thermal energy density of the quiescent coronal plasma. The most dominant source of energy in the corona is the magnetic field. The current consensus is that solar flares represent a process of liberating the magnetic energy stored in the corona.

The existence of the magnetic field in the corona does not necessarily mean that the magnetic energy is available there to heat the plasma. The electric currents that create the magnetic field are the ultimate source of energy. When no electric currents exist in the corona, the magnetic field in the corona is totally due to the currents flowing in the lower layers of the solar atmosphere (i.e., in the photosphere and below). Such magnetic fields are said to be free from distortion and have no energetic contribution to the phenomena in the corona. When the currents are induced in the corona, the magnetic field there is distorted, and contains the excess energy that can be liberated as a flare.

When an active region is born, there might be transient processes of energy liberation associated with the emergence of magnetic flux. After the initial relaxation is over, flare-productive active regions continue to grow and develop a highly distorted magnetic field configuration. This energy build-up phase will be regarded as a slow evolution of magnetic equilibria. This phase is followed by an explosive liberation of the stored magnetic energy, namely a flare.

The magnetic field in the corona is anchored in the dense photosphere and is controlled (passively moved around) by the flow of gas in the photosphere. Such a situation may be modeled by taking the photosphere as the lower boundary on which the boundary conditions can be specified. Therefore, the slow build-up of magnetic energy in the corona reflects the slow change in the boundary conditions near the photosphere. The energy release will be related to some sort of instability in the distorted magnetic field configuration. Although there can be other possibilities concerning the 
basic scenario of the flare process, in this review we will look into this canonical view in detail.

\section{Instability vs Non-Equilibrium}

Stability of a magnetic equilibrium can be most easily discussed by using an analogy with the equilibrium of a ball placed on a slope (Figure 1). The shape of the slope reflects an environment of the magnetic configuration, and may deform according to the change in the boundary condition for example.

The state $O$ is a stable equilibrium. As it evolves to $A_{1}$, the system becomes marginally stable. At $A_{2}$, the curve becomes slightly convex and the system is now unstable. This instability is 'weak', because the system will evolve into a neighboring stable equilibrium, without any drastic process.
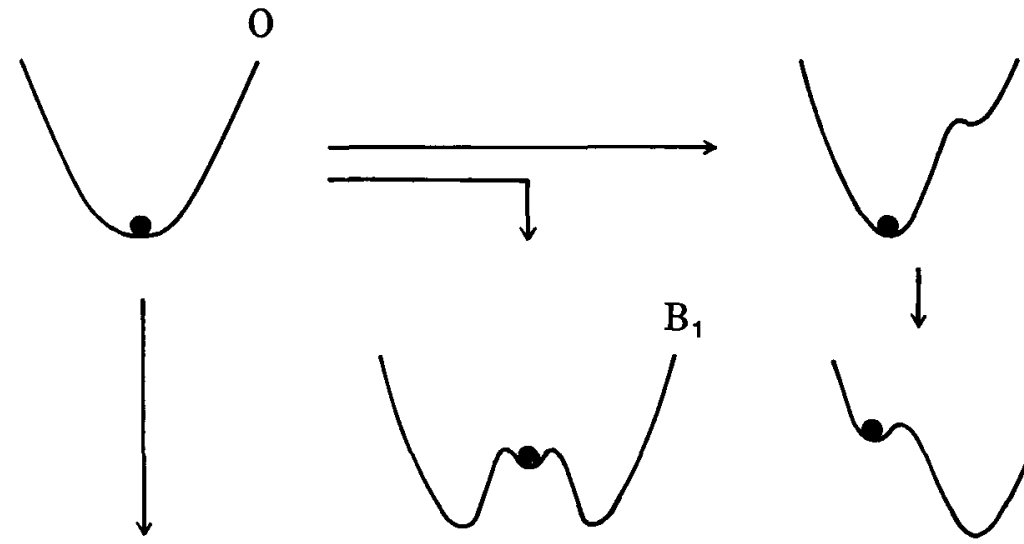

$\mathrm{C}_{1}$
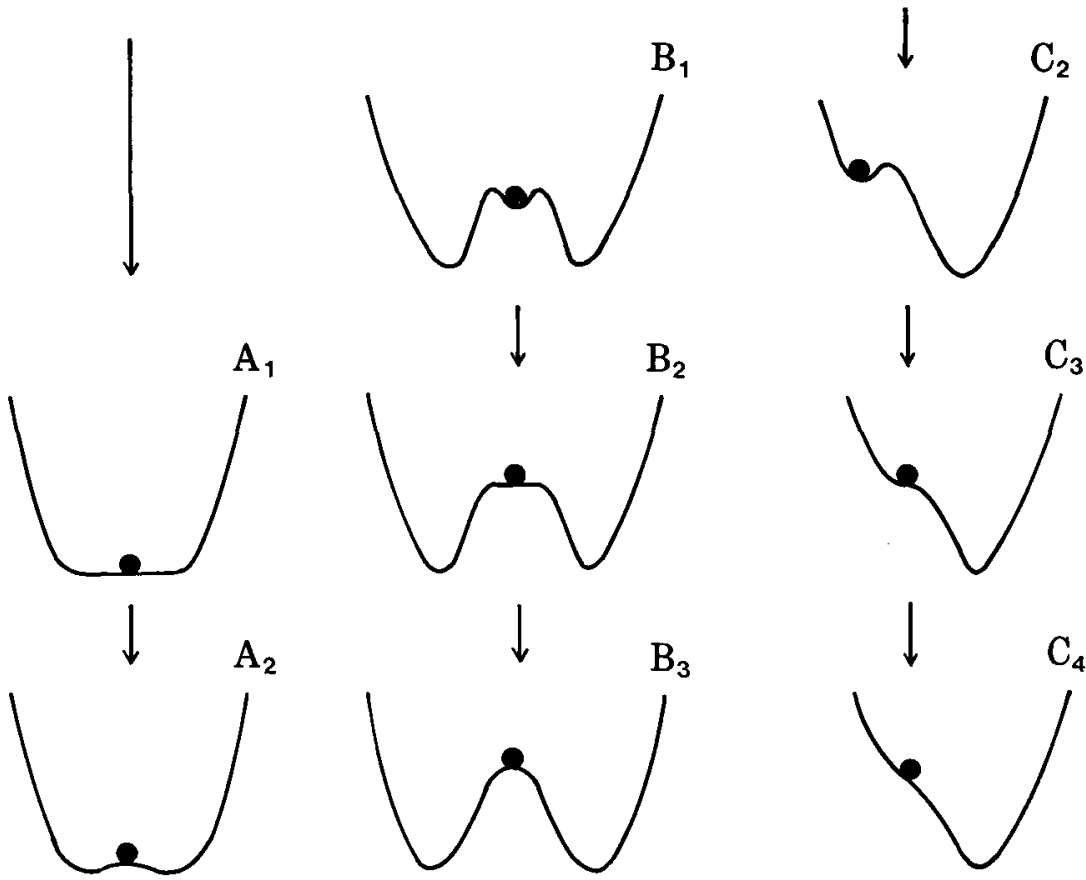

Fig. 1. Schematic representation of equilibrium sequences. The state $O$ is the initial stable equilibrium. Sequence $A$ leads to a weak instability, and sequence $B$ ends up with an explosive instability. Sequence $C$ leads to the loss of equilibrium. 
The state $B_{1}$ is called a meta-stable equilibrium, because it is stable against small amplitude perturbations but is unstable to large amplitude perturbations (Sturrock, 1966). At $B_{2}$ the system is marginally stable. The state $B_{3}$ is 'explosively unstable', because there is no stable equilibrium in the neighborhood of the original (unstable) equilibrium and the system undergoes a jump of finite amplitude.

The state $C_{2}$ is meta-stable and $C_{3}$ is marginally stable. As the system evolves to $C_{4}$, the equilibrium (i.e., the plateau in $\dot{C}_{3}$ ) is lost and the system exhibits a finite-amplitude jump toward a new equilibrium.

One notable difference between the explosive instability (sequence $B$ ) and the loss of equilibrium (sequence $C$ ) is that the resulting perturbation that grows is unidirectional in sequence $C$. In sequence $B$ the direction in which the system moves depends on the initial (infinitesimal) perturbation.

\section{Two-Dimensional Equilibria}

\subsection{BASIC EQUATION}

In order to study instabilities and disequilibrium of magnetic field configurations discussed above, we will next consider two-dimensional equilibria as an example (Birn and Schindler, 1981; Low, 1982). The $x$-coordinate is supposed to be an ignorable coordinate, and the magnetic field $\mathbf{B}$ is a function of $y$ and $z$ in the form

$$
\mathbf{B}=\left(B_{x}, \frac{\partial A}{\partial z},-\frac{\partial A}{\partial y}\right),
$$

where $A$ is a function of $y$ and $z$, and $B_{x}$ is a function of $A$. If the gas is stratified isothermally, the gas pressure $p$ must be written as

$$
p=p_{0}(A) \exp (-z / H),
$$

where $H$ is the (constant) scale height. The vector potential $A$ is determined by the so-called Grad-Shafranov equation

$$
\nabla^{2} A+\frac{1}{2} \frac{\partial}{\partial A}\left\{B_{x}^{2}+8 \pi p_{0} \exp (-z / H)\right\}=0 .
$$

The current-free state is represented by $\nabla^{2} A=0$, so that the second term reflects the distortion in the magnetic field. Therefore, we write

$$
\nabla^{2} A+\lambda F(A, z)=0
$$

and the field configuration is increasingly distorted as $\lambda$ is varied from zero to larger values. 
The boundary condition for $A$ is given at the $z=0$ plane (the photosphere) as $A=A_{b}$. By defining $A^{\prime}=A-\bar{A}$ with

$$
\nabla^{2} \bar{A}=0, \quad \bar{A}=A_{b} \quad \text { at } \quad z=0,
$$

the equation is reduced to a canonical form

$$
-\nabla^{2} A^{\prime}=\lambda F\left(\bar{A}+A^{\prime}, z\right), \quad A^{\prime}=0 \quad \text { at } \quad z=0 .
$$

\subsection{Multiplicity in ThE SOlutions}

At this point it is useful to compare this equation with the equation describing the equilibrium of a membrane, namely,

$$
-\tau \nabla^{2} \xi=\sigma g .
$$

Here $\tau(\xi)$ is the tension, $\sigma$ is the surface density, $g$ is the gravitational acceleration, and $\xi$ is the displacement in the membrane which vanishes on the boundary. (We have slightly deviated from the linearization assumption that led to Equation (7) and introduced the $\xi$-dependence in $\tau$.) We find the following correspondence:

$$
\xi \leftrightarrow A^{\prime}, \quad \tau \leftrightarrow 1 / F, \quad \sigma g \leftrightarrow \lambda .
$$

We can see that larger $\lambda$ corresponds to heavier mass loading in the membrane. The larger $F$ is, the more fragile the membrane is.

We may roughly set $-\nabla^{2} \rightarrow 1 / L^{2}$, where $L$ stands for the size of the system. Then Equations (4) and (7) are simplified as

$$
\lambda F=A^{\prime} / L^{2}
$$

and

$$
\sigma g / \tau=\xi / L^{2}
$$

respectively, and the multiplicity in the solutions can be argued graphically.

Figure 2(a) shows that the solution exists and is unique when $\partial F / \partial A<0$. On the other hand Figure 2(b) reveals that if $F>0, \partial F / \partial A>0$, and $\partial^{2} F / \partial A^{2}>0$, no solution exists for $\lambda>\lambda_{*}$ but two (or more) solutions exist for $\lambda<\lambda_{*}$. (Similar argument holds if $F<0, \partial F / \partial A>0$, and $\partial^{2} F / \partial A^{2}<0$.)

Figure 2(c) is equivalent to Figure 2(b) but in a different format. The curve in Figure 2(c) represents the restoring force in the membrane as a function of the displacement $\xi$. The solution with a smaller magnitude of $\xi$ (designated as the branch I solution) is stable. The branch II solution will be unstable because slightly larger displacement $\xi$ leads to smaller restoring force. The solution at $\lambda=\lambda_{*}$ represents the critical equilibrium and is marginally stable. These stability properties are for the cases in which the perturbations given to the system are also two-dimensional. If threedimensional perturbations are considered, the branch I solutions may be unstable.

If the function $F$ satisfies $\partial F / \partial A<0$, the solution formally exists for an arbitrary value of $\lambda$. However, the solution sequence terminates physically at some $\lambda$, beyond which the 


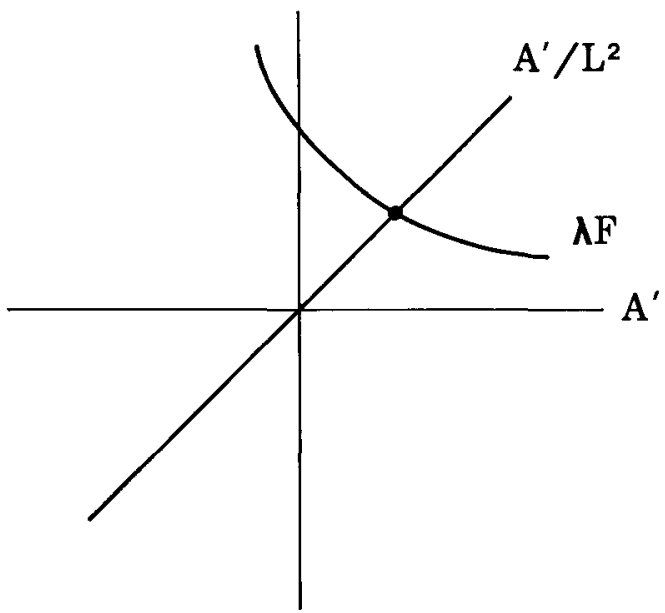

Fig. 2a. Schematic diagram showing the uniqueness in the solutions to Equation (8) when $\partial F / \partial A<0$.

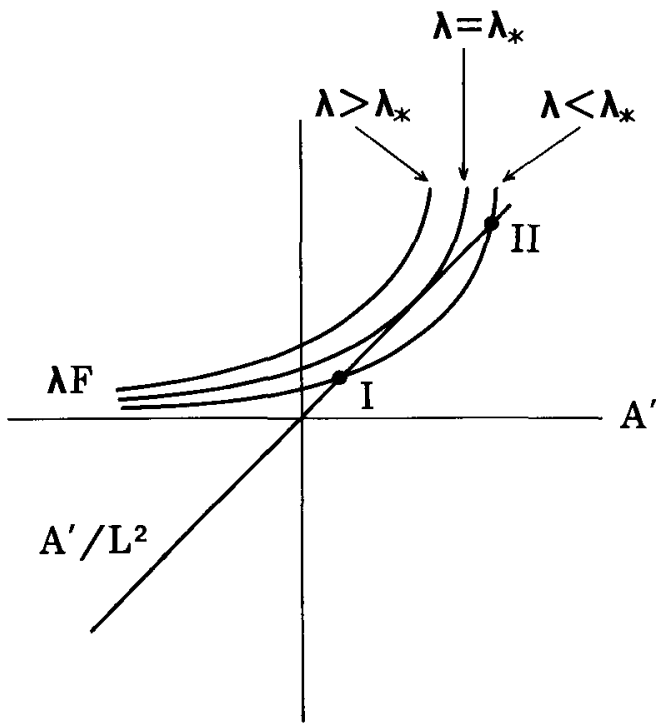

Fig. 2b. Schematic diagram showing the multiplicity in the solutions to Equation (8) when $\partial F / \partial A>0$.

quantity $B_{x}^{2}+8 \pi p_{0} \exp (-z / H)$ goes to negative. In the following we will concentrate on the cases with multiple solutions, because of their relevance to the flare models.

\subsection{LOSS OF EQUILIBRIUM BY INCREASING SHEAR}

First we will discuss the case with $p=0$, so that $\lambda F$ measures the effect of $B_{x}$. The presence of $B_{x}$ makes the field lines tilted from the $y z$-plane. The separation between the two footpoints $(\Delta x)$ is called the shear. The variation of $\Delta x$ as a function of $\lambda$ is as in Figure 3. Solutions may be lost if $\lambda$ is driven beyond $\lambda_{*}$. 


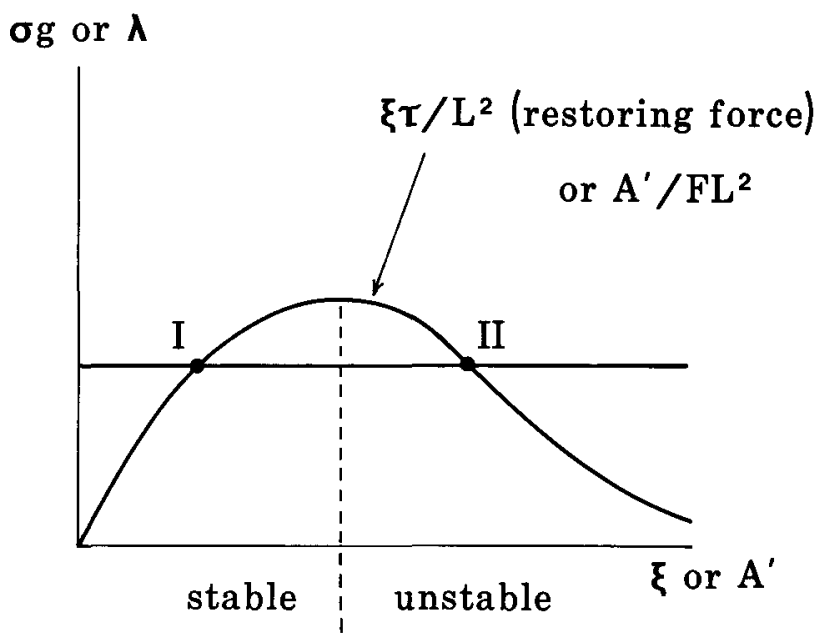

Fig. 2c. Same as Figure 2(b) but in a different format. The ordinate corresponds to the equivalent restoring force.

However, it is not $B_{x}$ but is $\Delta x$ that directly represents the effect of boundary motions deforming the magnetic configuration. If $\Delta x$ is taken as the control parameter, then $\lambda$ varies from zero to $\lambda_{*}$ and then decreases along the branch II as $\Delta x$ increases. This behavior is explained by Jockers (1976) as follows. The shear $\Delta x$ is proportional to $B_{x} \times L_{F}$, where $L_{F}$ is the length of the field line. Along branch I solutions, $L_{F}$ is nearly constant and $B_{x}$ is proportional to $\Delta x$. On branch II the increase in $\Delta x$ is accounted for by the increase in $L_{F}$, in spite of the decrease in $B_{x}$ or $\lambda$.

Aly (1985) derived the integral inequality

$$
\int B_{x}(A) \Delta x(A) \mathrm{d} A \leqq\left[\int B_{z}^{2} \mathrm{~d} y \int y^{2} B_{z}^{2} \mathrm{~d} y\right]^{1 / 2}
$$

and found that $B_{x} \rightarrow 0$ as $\Delta x \rightarrow \infty$. He interpreted this as the asymptotic formation of current sheets as $\Delta x$ becomes very large.

The implication of the two arguments above is that the equilibrium will not be lost as the shear $\Delta x$ increases. The solutions with very large shear may, however, belong to the branch II and will be unstable against two-dimensional perturbations. The stability of the equilibria with respect to three-dimensional perturbations will be discussed later.

\subsection{LOSS OF EQUILIBRIUM BY INCREASING PRESSURE}

Next we will look into the case with $B_{x}=0$, so that $\lambda F$ is solely due to the gas pressure. Then $\lambda$ (namely pressure) is a natural control parameter, and there would be no reason to exclude the cases with $\lambda>\lambda_{*}$. Therefore, the equilibrium will be lost when $\lambda$ is driven beyond $\lambda_{*}$. Low (1981) argued that such disequilibrium may occur in two ways. One is when the prescribed pressure is too high to be magnetically confined. The effect of gravity is not essential in this case. The other case is due to the magnetic buoyancy. 

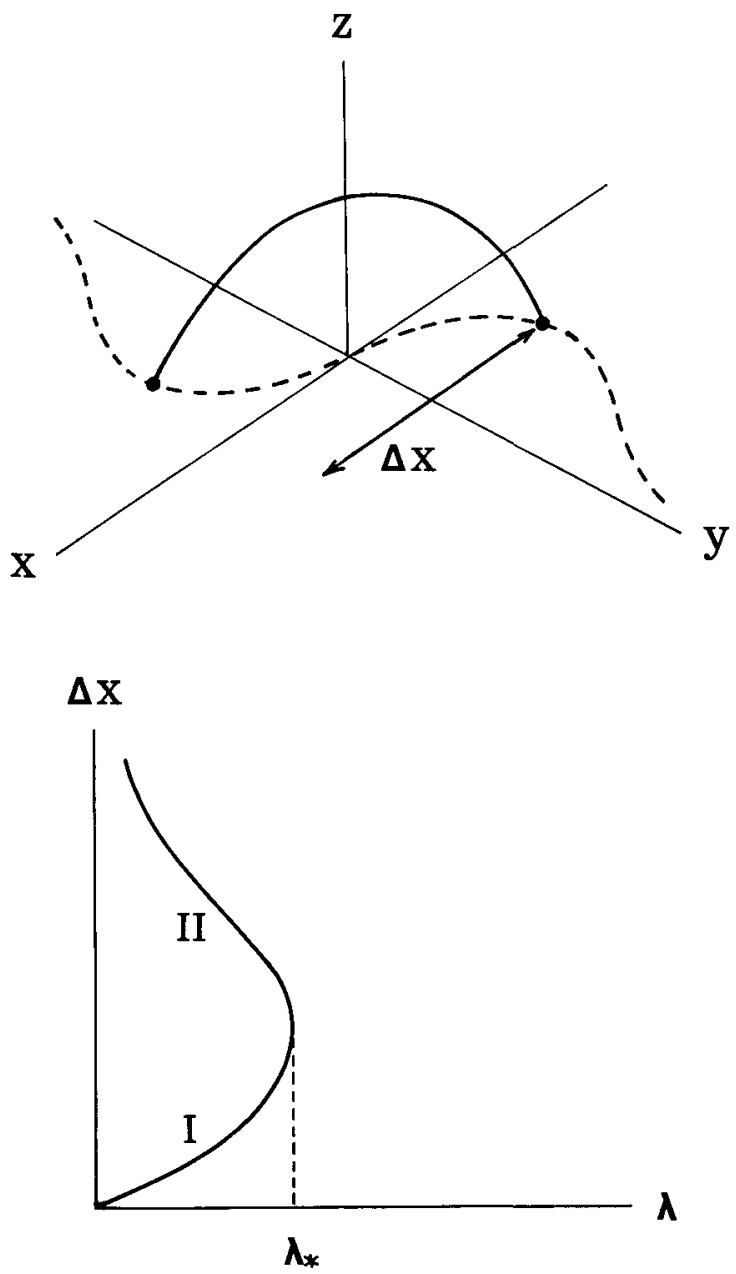

Fig. 3. Definition of the shear $\Delta x$ (top) and the behavior of $\Delta x$ as a function of the parameter $\lambda($ bottom).

Zwingmann (1987) performed the numerical simulations including both the shear and the pressure effects, and found that the catastrophe requires finite pressure but can take place without shear. Therefore, the presence of finite pressure is necessary for the loss of equilibrium. However, shearless configurations may well be susceptible to the interchange instability (Section 4.2) even if $\lambda<\lambda_{*}$, when three-dimensional perturbations are considered. It is, therefore, debatable whether pressure-dominated equilibria can reach the critical state $\lambda=\lambda_{*}$ before getting unstable.

\section{Ideal MHD Instabilities}

When the effect of resistivity is negligible (ideal MHD), instabilities that can take place may be divided into two classes. One is the kink instability, driven by the Lorentz force. 
This instability may occur even with the absence of the pressure. The other is the interchange instability, driven by the pressure force.

\subsection{KinK INSTABILITy OF LOOPS}

Kink instability appears in cylindrical loops as a growing helical perturbation. Anzer (1968) showed that infinitely long loops are always unstable to the kink instability. In actuality the loop has a finite length, and its footpoints are rooted in the dense photosphere. Therefore, the displacements are required to vanish at two footpoint cross sections. This effect of 'line-tying' tends to stabilize the kink instability (Raadu, 1972). For example the constant-pitch force-free field in cylindrical coordinates $(r, \theta, z)$,

$$
\begin{aligned}
& B_{z}=B_{0} /\left[1+r^{2} / b^{2}\right]^{-1}, \\
& B_{\theta}=(r / b) B_{z} \quad(b=\text { const. }),
\end{aligned}
$$

is stable if the length of the loop $L$ is such that $L / b \lesssim 2.5 \pi$. That is, this loop is stable if the twist over the length of the loop does not exceed 1.25 turn (Hood and Priest, 1981).

The nonlinear development of the kink instability will be described by the equation for the mode amplitude $\xi$ as

$$
\frac{\partial^{2} \xi}{\partial t^{2}}=\gamma^{2} \xi+\delta \xi^{3}+\cdots
$$

Even when the system is linearly unstable $\left(\gamma^{2}>0\right)$, nonlinear effects stabilize the instability if $\delta<0$. On the other hand if $\delta>0$, the nonlinear effects enhance the instability, leading to the explosive instability. For example, in the case of a sharp boundary pinch (plasma in $r<r_{1}$, vacuum in $r_{1}<r<r_{2}$, with a current sheet at $r=r_{1}$ and a conducting wall at $r_{2}$ ), $\delta$ is found to be

$$
\delta \sim \frac{\left(r_{2} / r_{1}\right)^{4}-2}{\left(r_{2} / r_{1}\right)^{4}-1},
$$

so that the kink instability is explosive if $r_{2} \gtrsim 1.2 r_{1}$ (Pao, 1978). The nonlinear development of the kink instability was studied by Sakurai (1976).

\subsection{Stability of ARCADES}

Roughly speaking, the magnetic arcade is equivalent to a straight magnetic loop sliced in half along the loop axis and placed on the photosphere. Helical perturbations that characterize the kink instability are prohibited in magnetic arcades due to the line-tying condition. If the effect of pressure is large enough, the arcade can be unstable to the interchange instability. Hood (1986) derived the sufficient condition for instability as

$$
\begin{aligned}
& \frac{B^{2}}{r^{2}}+\frac{\pi^{2}}{4} B_{x}^{2}\left(\frac{1}{q} \frac{\mathrm{d} q}{\mathrm{~d} r}\right)^{2}+\frac{2}{r} \frac{\mathrm{d} p}{\mathrm{~d} r}+\frac{16 \pi \Gamma p B_{\theta}^{2}}{r^{2}\left(4 \pi \Gamma p+B^{2}\right)}<0 \\
& \left(q=r B_{x} / B_{\theta}\right) .
\end{aligned}
$$


Here $\Gamma$ denotes the ratio of specific heats, and the cylindrical coordinates $(r, \theta)$ around the $x$-axis are taken as in Figure 4(a). The equilibrium quantities are functions of $r$. The instability arises when the plasma confined by the magnetic field $(\mathrm{d} p / \mathrm{d} r<0)$ escapes from the confinement by making thin sheets intruding through the magnetic field. Stabilizing effects come from varying pitch of the field lines (the second term) and from the line tying (the first and the fourth terms). For force-free arcades $(p=0)$ this equation does not suggest instability, and a higher-order analysis is necessary to determine the stability. Hood and Anzer (1987) studied the stability of several force-free arcade configurations and could not find any unstable modes. However, if the configuration contains closed field lines detached from the photosphere (the magnetic island, Figure 4(b)), which might be related to dark filaments on the Sun (Anzer and Tandberg-Hanssen, 1970), the situation is similar to a loop surrounded by an external magnetic field and the rigid boundary (i.e., the photosphere). Hood and Priest (1980) showed that if the height of the closed loop above the photosphere is sufficiently large, the loop may be unstable against the kink instability.

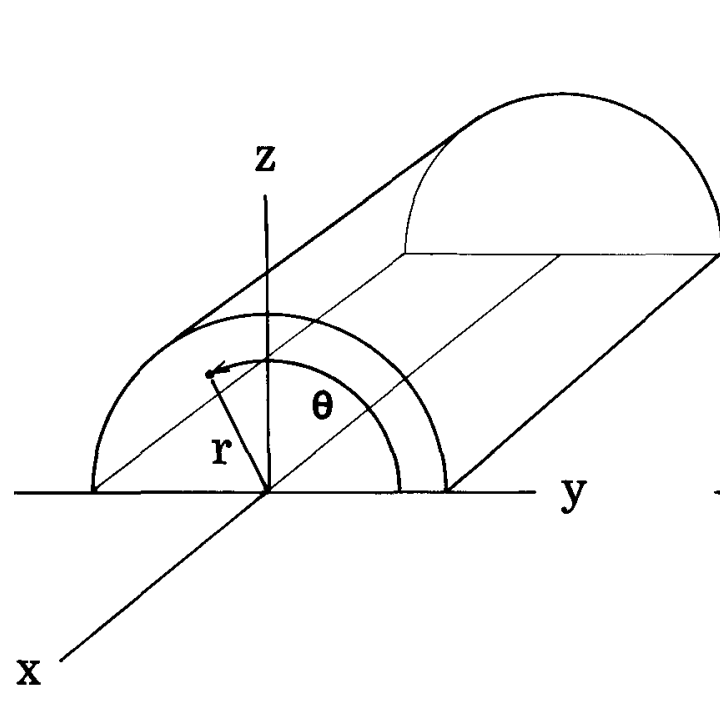

a

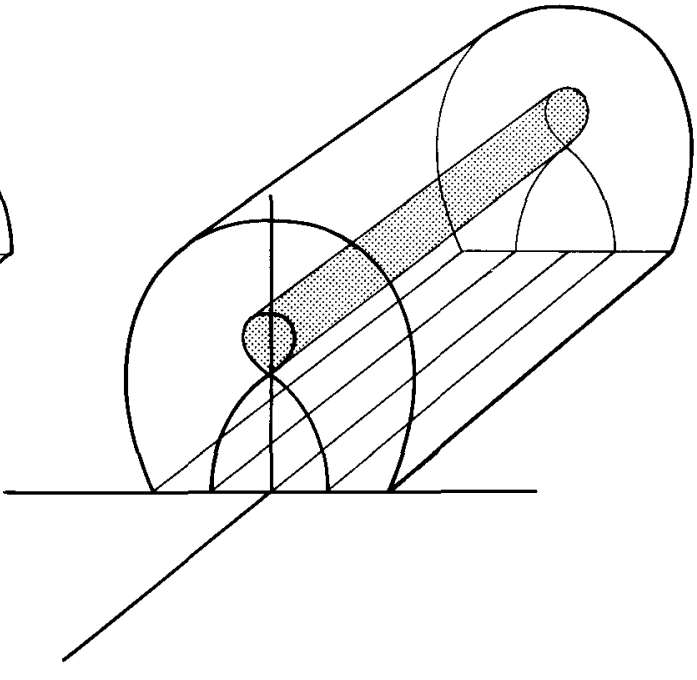

b

Fig. 4. (a) The magnetic arcade configuration. (b) The arcade with a magnetic island or 'a filament' (shaded).

\section{Non-Ideal Effects}

Deviation from the ideal MHD situation can be found in various ways. The effect of non-zero resistivity is to violate the frozen-in condition of lines of force, and to modify the energy equation by Joule heating. Non-adiabatic effects may also come from the heat conduction and radiative processes. 


\subsection{Finite RESISTIVITY}

A well-known instability in the presence of a finite resistivity is the tearing instability (Furth, Killeen, and Rosenbluth, 1963). Let us denote the dynamical and resistive time-scales by $\tau_{\mathrm{A}}$ and $\tau_{\mathrm{R}}$, respectively. They are defined as

$$
\tau_{\mathrm{A}}=L / V_{\mathrm{A}}, \quad \tau_{\mathrm{R}}=L^{2} / \eta,
$$

where $L$ is the size of the system, $V_{\mathrm{A}}$ is the Alfven speed, and $\eta$ is the resistivity. The magnetic Reynolds number $S$ is defined by $S=\tau_{\mathrm{R}} / \tau_{\mathrm{A}}$, which is much larger than unity in the solar atmosphere. The growth rate of the tearing instability is expressed as

$$
\gamma \sim S^{\alpha} / \tau_{\mathbf{R}}
$$

where $\alpha$ ranges from $\frac{2}{5}$ to $\frac{2}{3}$ (Steinolfson and Van Hoven, 1983).

Another important process is the magnetic reconnection. Original analytic but simplified treatment (Petschek, 1964) assumed a steady state, but recent progress has been made by time-dependent numerical simulations. An important implication derived from them is that the rate of reconnection can be as fast as one likes, if the flow field is set up which forces the reconnection to occur (Sato and Hayashi, 1979). Then the question is how such a favorable flow is set up.

In this respect it is adequate to mention the so-called coalescence instability (Finn and Kaw, 1977). This instability takes place, for example, when two loops with parallel electric currents attract with each other. Until two loops come in contact and merge, the process is described essentially as an ideal MHD process. The explosive nature of this instability is demonstrated by using a self-similar scaling (Sakai and Tajima, 1986). In one-dimensional coalescence in the $x$-direction, they found the velocity $v$, the density $\rho$, and the magnetic field $B$ to scale as

$$
\begin{aligned}
& v=\frac{1}{a} \frac{\mathrm{d} a}{\mathrm{~d} t} x, \\
& \rho=\frac{\rho_{0}}{a},
\end{aligned}
$$

and

$$
B=B_{0} \frac{x}{x_{0}} \frac{1}{a^{2}} .
$$

Here $\rho_{0}, B_{0}$, and $x_{0}$ are constants, and the scale factor $a$ varies as $a \sim\left(t_{0}-t\right)^{2 / 3}$. The time $t_{0}$ represents the epoch when two current systems collide.

It may well be that not only the coalescence instability but also other MHD instabilities are able to provide a driving force which promotes a rapid reconnection. For example the tearing instability shows faster growth ( $\alpha$ in Equation (16) is close to $\frac{2}{3}$ ) when it is driven externally (Spicer and Brown, 1981). 


\subsection{Thermal instaBilities}

Thermal instabilities may arise in several ways. If the radiative cooling rate decreases as the temperature increases, there will be a thermal runaway (Field, 1965). The time-scale of this radiative instability is

$$
\tau_{\mathrm{rad}}=\frac{3 n k T}{n^{2} Q_{\mathrm{rad}}(T)},
$$

where $n$ is the number density of the plasma and $Q_{\mathrm{rad}}(T)$ is the radiative output power per unit number density at temperature $T$.

Similarly if the Joule heating rate increases as the temperature increases, the plasma is unstable to the so-called Joule heating mode (Heyvaerts, 1974). This instability arises because

$$
T \uparrow \rightarrow \eta \sim T^{-3 / 2} \downarrow \rightarrow j \uparrow(E \sim \text { const. }) \rightarrow \text { heating } \uparrow \rightarrow T \uparrow .
$$

For the electric field $E$ to stay constant, the time-scale of this sequence must be as long as $\tau_{\mathbf{R}}$. For faster time-scales the decrease in $\eta$ will lead to the decrease in $E$ while the current $j$ is kept constant, and the heating is reduced instead.

In the solar corona the ordering $\tau_{\mathrm{A}}<\tau_{\mathrm{rad}}<\tau_{\mathrm{R}}$ holds. The interplay between thermal and tearing instabilities was studied, e.g., by Steinolfson (1983). When the radiation is unstable, there are cases in which the radiative instability grows faster than the tearing instability. The radiative instability in such a case involves the reconnection of magnetic fields as well. Joule heating mode does not appear unless the radiative energy loss is artificially suppressed.

Thermal instabilities can play a role in setting up a pre-flare current filamentation or in triggering other more energetic instabilities. It seems unlikely, however, that they serve as the primary mechanism of flare energy release.

\subsection{Thermal STABILITY OF CORONAL LOOPS}

Thermal stability of coronal loops is more complicated than the argument given above, due to the coupling between the coronal loop and the chromosphere/photosphere at its footpoints. The treatment of the mass flow into or out of the loop has yielded contradictory results. McClymont and Craig (1985) claimed that the coronal loops are stable, while Antiochos et al. (1985) found instabilities. Martens and Kuin (1983) suggested that the loops we see are not static but are exhibiting a limit cycle behavior. Recent analysis (Klimchuk, Antiochos, and Mariska, 1987) indicated that the stability of coronal loops critically depends on the height or the length of loops. Apparently more study is needed to resolve this important issue.

\section{Summary}

A possible sequence from a current-free (non-distorted) magnetic field configuration to an explosive energy release is shown in Figure 5. 

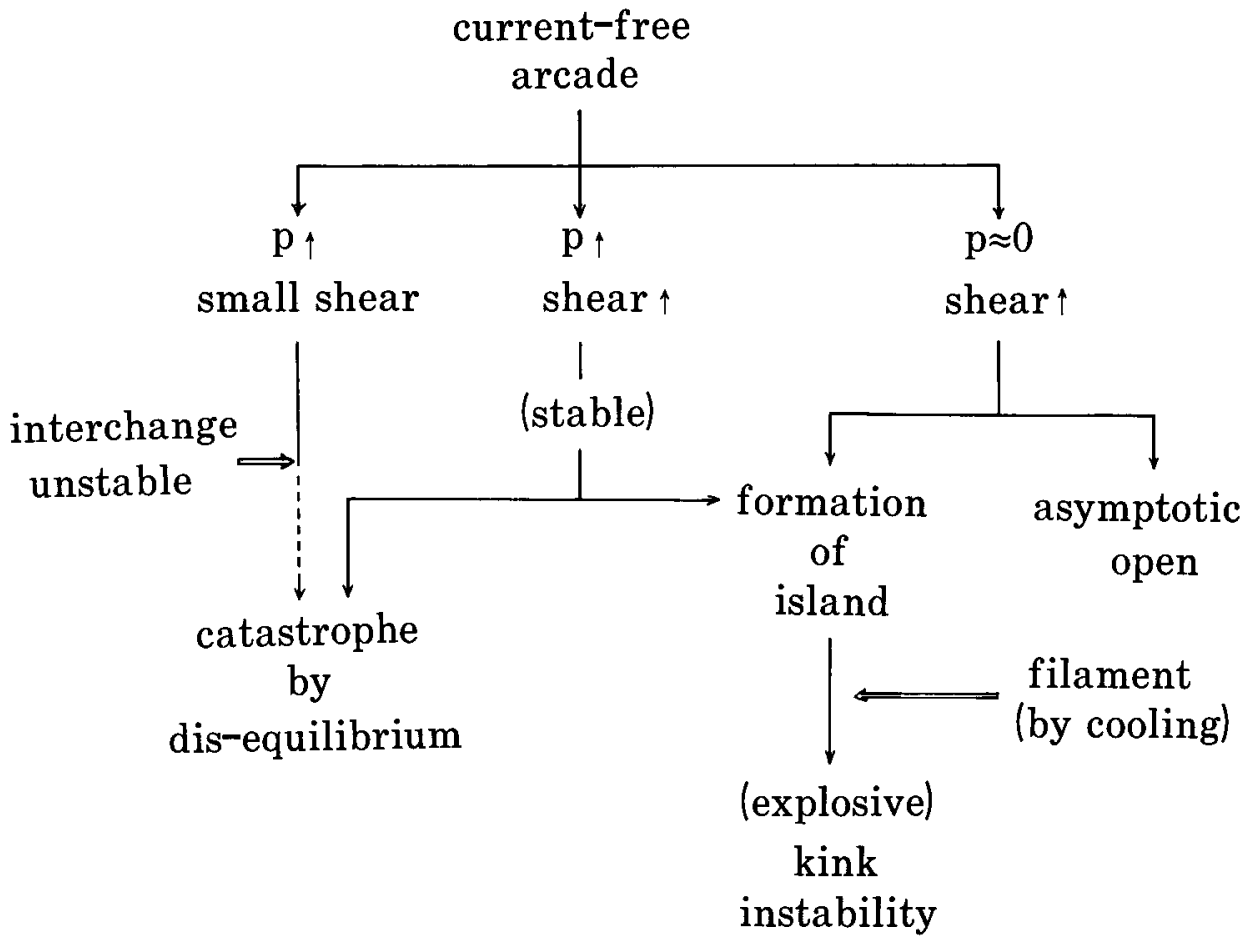

Fig. 5. Various possibilities which may lead to catastrophic energy release, starting from an initial stable equilibrium.

(a) If the pressure increases in an arcade with small magnetic shear, 2-D equilibrium sequence models predict a catastrophe by disequilibrium. However, it is likely that the system may become unstable to the interchange instability before the catastrophe. [The interchange instability is here assumed to be a local, non-catastrophic instability. This may not be true if the magnetic reconnection is involved (Parker, 1973; Uchida and Sakurai, 1977).]

(b) If the pressure increases in a sheared arcade, the system may remain stable against the interchange instability until the system loses the equilibrium. However, the requirement of a large pressure in cases (a) and (b) is unsatisfactory, in considering the magnetically dominated condition in the corona. Therefore, the case (c) below is concluded to be the most promising.

(c) If the shear increases in a nearly force-free arcade, there is evidence that the equilibrium always exists and the magnetic configuration asymptotically approaches the open magnetic field. Force-free arcades are highly stable, with the absence of a magnetic island. The configuration with the magnetic island can become unstable against the kink instability.

It is premature to say that any force-free arcades without magnetic islands are stable. Nevertheless it is suggestive that the presence of the magnetic island in an arcade could be crucial in considering the stability of the system. Physically speaking, the magnetic 
island may correspond to a filament if cool material is present there. In some pressuredominated cases (Melville, Hood, and Priest, 1987), the island forms before the catastrophic point is reached. In all the force-free arcade models available up to now, the island only forms at or after the critical point is reached (Low, 1977; Jockers, 1978). It is not clear whether this is a general rule or because only a small number of examples were studied. Therefore, the important issues raised here are (i) to thoroughly study the stability of force-free arcades, and (ii) to clarify whether the magnetic island can form in stable force-free equilibrium sequences.

In the models developed so far, the island either emerges from the bottom boundary (Low, 1977; Jockers, 1978; Melville, Hood, and Priest, 1987) or forms at some height in the volume $z>0$ (Zwingmann, 1987). The latter case physically requires the magnetic reconnection (Anzer and Priest, 1985). When the magnetic island (or a magnetic loop) is formed in an arcade, it may undergo explosive kink instability. This is in accord with the fact that flares are often associated with the activation of filaments. The role played by the cool material which comprises the filament is not clear, however. It might be that an invisible filament, namely a magnetic island not filled with cool material, can play the same role.

The scenario described above may correspond to the so-called two-ribbon flares, which are in contrast with the simple loop flares (Priest, 1981). For the latter class of flares, they could be due to the kink-unstable loops which may form simply by twisting the existing coronal loops. The magnetic reconnection (or the tearing instability) driven by explosive MHD instabilities may, therefore, be a possible explanation for both types of flares. This conjecture does not exclude the possibility of other important classes of flare models such as the emerging flux model (Heyvaerts, Priest, and Rust, 1977).

\section{References}

Aly, J. J.: 1985, Astron. Astrophys. 143, 19.

Antiochos, S. K., Shoub, E. C., An, C.-H., and Emslie, A. G.: 1985, Astrophys. J. 298, 876.

Anzer, U.: 1968, Solar Phys. 3, 298.

Anzer, U. and Priest, E. R.: 1985, Solar Phys. 95, 263.

Anzer, U. and Tandberg-Hanssen, E.: 1970, Solar Phys. 11, 61.

Birn, J. and Schindler, K.: 1981, in E. R. Priest (ed.), Solar Flare Magnetohydrodynamics, Gordon and Breach, London, p. 337.

Field, G. B.: 1965, Astrophys. J. 169, 379.

Finn, J. M. and Kaw, P. K.: 1977, Phys. Fluids 20, 72.

Furth, H. P., Killeen, J., and Rosenbluth, M. N.: 1963, Phys. Fluids 6, 459.

Heyvaerts, J.: 1974, Astron. Astrophys. 37, 65.

Heyvaerts, J., Priest, E. R., and Rust, D. M.: 1977, Astrophys. J. 216, 123.

Hood, A. W. and Anzer, U.: 1987, Solar Phys. 111, 333.

Hood, A. W. and Priest, E. R.: 1980, Solar Phys. 66, 113.

Hood, A. W. and Priest, E. R.: 1981, Geophys. Astrophys. Fluid Dyn. 17, 297.

Hood, A. W.: 1986, Solar Phys. 103, 329.

Jockers, K.: 1976, Solar Phys. 50, 405.

Jockers, K.: 1978, Solar Phys. 56, 37.

Klimchuk, J. A., Antiochos, S. K., and Mariska, J. T.: 1987, Astrophys. J. 320, 409.

Low, B. C.: 1977, Astrophys. J. 212, 234.

Low, B. C.: 1981, Astrophys. J. 251, 352. 
Low, B. C.: 1982, Rev. Geophys. Space Phys. 20, 145.

Martens, P. C. H. and Kuin, N. P. M.: 1983, Astron. Astrophys. 123, 216.

McClymont, A. N. and Craig, I. J. D.: 1985, Astrophys. J. 289, 820.

Melville, J. P., Hood, A. W., and Priest, E. R.: 1987, Geophys. Astrophys. Fluid Dyn. $39,83$.

Pao, Y.: 1978, Phys. Fluids 21, 765.

Parker, E. N.: 1973, Astrophys. J. 180, 247.

Petschek, H. E.: 1964, in W. N. Hess (ed.), NASA Symposium on the Physics of Solar Flares, p. 425.

Priest, E. R.: 1981, in E. R. Priest (ed.), Solar Flare Magnetohydrodynamics, Gordon and Breach, London, p. 2.

Raadu, M. A.: 1972, Solar Phys. 22, 425.

Sakai, J. and Tajima, T.: 1986, ESA SP-251, 77.

Sakurai, T.: 1976, Publ. Astron. Soc. Japan 28, 177.

Sato, T. and Hayashi, T.: 1979, Phys. Fluids 22, 1189.

Spicer, D. S. and Brown, J. C.: 1981, in S. Jordan (ed.), The Sun as a Star, NASA SP-450, p. 413.

Steinolfson, R. S.: 1983, Phys. Fluids 26, 2590.

Steinolfson, R. S. and Van Hoven, G.: 1983, Phys. Fluids 26, 117.

Sturrock, P. A.: 1966, Phys. Rev. Letters 16, 270.

Uchida, Y. and Sakurai, T.: 1977, Solar Phys. 51, 413.

Zwingmann, W.: 1987, Solar Phys. 111, 309. 